\title{
On the zeros of $\zeta^{\prime}(s)-a$
}

by

R. Balasubramanian (Madras) and K. Ramachandra (Bombay)

1. Introduction and notation. As usual we write $s=\sigma+i t$. The main object of this paper is to prove the following

THEOREM 1. Let $a$ be any complex constant and $\mu$ any real constant satisfying $0<\mu<1$. Then there exists a constant $\delta=\delta(a, \mu)(>0)$ depending only on a and $\mu$ such that the number of distinct zeros of $\zeta^{\prime}(s)-a$ in $\left(\sigma \geq 1 / 2+\delta, T \leq t \leq T+T^{\mu}\right)$ exceeds a positive constant multiple of $T^{\mu}$. (Hereafter we write $\gg T^{\mu}$ to mean this.)

Remark. The history of the theorem on the zeros of $\zeta^{\prime}(s)-a$ is connected with the names B. C. Berndt [6] (see p. 287 of [8]) and N. Levinson and H. L. Montgomery [7] (see pp. 287-289 of [8]). See also Theorem 11.5(C) on p. 298 of [8] for the earlier history. But our Theorem 1 deals with the distribution of the zeros of $\zeta^{\prime}(s)-a$ in "short $t$-slabs" in the "right half of the critical strip" (i.e. the region $\sigma \geq 1 / 2+\delta$ ), as compared with earlier results. Another point about our proof of Theorem 1 is its novelty and its generality.

We will be concerned with proving a similar theorem for more general functions $G(s)$ defined for large $\sigma$ by a convergent series $1-\sum_{n=2}^{\infty} b_{n} \mu_{n}^{-s}$ where $\mu_{2}, \mu_{3}, \ldots$ are real numbers and $b_{2}, b_{3}, \ldots$ are complex numbers restricted by the following conditions:

(i) Put $\mu_{1}=1$. Then for $n=1,2,3, \ldots, C_{1}^{-1} \leq \mu_{n+1}-\mu_{n} \leq C_{1}$ where $C_{1}(\geq 1)$ is any fixed constant,

(ii) $G(s)$ can be continued analytically in $\left(\sigma \geq 1 / 2, T \leq t \leq T+T^{\mu}\right)$ and there the maximum of $|G(s)|$ is $\leq T^{C_{2}}$ where $C_{2}(>0)$ is any fixed constant, and

(iii) $\left|b_{n}\right|<n^{C_{3}}$ where $C_{3}(>0)$ is any constant.

Theorem 1 is a special case of

THEOREM 2. Let $\mu_{n}=\left(n_{0}+n-1\right) n_{0}^{-1}$ where $n_{0}$ is any integer constant $\geq 1$. Let $\sum_{n=2}^{\infty}\left|b_{n}\right|^{2} n^{-1-\varepsilon}$ be convergent for every $\varepsilon>0$ and $\rightarrow \infty$ as $\varepsilon \rightarrow 0$. 
Let further

$$
\lim _{\varepsilon \rightarrow 0} \inf \min _{q \geq 2}\left\{q^{2}\left(\sum_{n=2}^{\infty}\left|b_{n}\right|^{2} n^{-1-\varepsilon}\right)^{1 / q}\left(\sum^{\prime}\left|b_{n}\right|^{2} n^{-1-\varepsilon}\right)^{-1}\right\}=0
$$

where the accent denotes the sum over all integers $\geq n_{0}+1$ for which $n_{0}+$ $n-1$ is prime. Then $G(s)$ has $\gg T^{\mu}$ distinct zeros in $(\sigma \geq 1 / 2+\delta$, $\left.T \leq t \leq T+T^{\mu}\right)$ for every fixed $\mu$ with $0<\mu<1$ and a suitable constant $\delta$ $(>0)$ depending only on $\mu, C_{1}, C_{2}$ and $C_{3}$.

Remark 1. Theorem 1 follows from the observations $\zeta^{\prime}(s)=$ $-2^{-s}(\log 2) G(s)$ for a suitable $G(s)$ and if $a \neq 0, \zeta^{\prime}(s)-a=-a G(s)$ for a suitable $G(s)$.

R e m ark 2. Obviously we can deduce similar theorems for higher derivatives of $\zeta(s)$. We can also get similar theorems for the derivatives of $\zeta$ and $L$-functions and for $\zeta$-functions of ray classes in any algebraic number field. However, we have neater theorems for $\zeta(s)-a(a \neq 0)$ and certain more general functions (compared with $\zeta(s)-a(a \neq 0))$. But these need a different treatment and are dealt with in [5].

For general $\mu_{n}$ we need a conjecture (see Section 2) which is true for example for $\mu_{n}=n+\sqrt{2}$. It is also true for more general situations. Thus we can state

THeOREM 3. Let $b_{2}, b_{3}, \ldots$ be non-negative real numbers and $\mu_{n}=n+\sqrt{2}$ $(n \geq 2)$. Then subject to the only conditions that $\sum_{n=2}^{\infty}\left|b_{n}\right|^{2} n^{-1-\varepsilon}$ shall be convergent for every $\varepsilon>0$ and that it shall tend to infinity as $\varepsilon \rightarrow 0, G(s)$ has $\gg T^{\mu}$ distinct zeros in $\left(\sigma \geq 1 / 2+\delta, T \leq t \leq T+T^{\mu}\right)$ for every constant $\mu(0<\mu<1)$ and a suitable positive constant $\delta$ depending only on $\mu, C_{1}$, $C_{2}$ and $C_{3}$.

Remark 1. This theorem is true for all those sequences $\mu_{n}$ for which the conjecture in Section 2 is true.

Remark 2. This theorem includes functions like

$$
1-\sum_{n=1}^{\infty} d(n)(n+\sqrt{2})^{-s}, \quad 1-\sum_{r}(N(r)+\sqrt{2})^{-s}
$$

where $r$ runs over all ideals in a ray class of an algebraic number field and so on.

As a closing remark in this section we mention that our method enables us to prove something stronger. For example in Theorems 1 and 3 we can prove that the number of zeros counted with multiplicity is either $\gg T^{\mu} \log T$ or there exist $\gg T^{\mu}$ distinct zeros of odd orders. For this we have to replace Lemma 1 of Section 3 by our Theorem 3 of [1]. 
2. Some preliminaries. In this section we collect together some results of our earlier papers and state a Local Convexity Theorem [3], a lower bound for the mean-value of Titchmarsh series [4] and two simple lemmas from [2]. We number these results with an asterisk thus: Theorems $1^{*}, 2^{*}, 3^{*}$ and $4^{*}$ and Lemmas $1^{*}$ and $2^{*}$. Also we state a conjecture.

(Local Convexity) Theorem $1^{*}$. Suppose $f(s)$ is an analytic function of $s=\sigma+i t$ defined in the rectangle

$$
R:\left\{a \leq \sigma \leq b, t_{0}-H \leq t \leq t_{0}+H\right\}
$$

where $a$ and $b$ are constants with $a<b$. Let the maximum of $|f(s)|$ taken over $R$ be $\leq M$. Let $a \leq \sigma_{0}<\sigma_{1}<\sigma_{2} \leq b$ and let $A$ be any large positive constant. Let $r$ be any positive integer, $0<D<H$ and $s_{1}=\sigma_{1}+i t_{0}$. Then $\left|2 \pi f\left(s_{1}\right)\right| \leq 2\left\{I_{0}^{\sigma_{2}-\sigma_{1}}\left(I_{2}+M^{-A}\right)^{\sigma_{1}-\sigma_{0}}\right\}^{\left(\sigma_{2}-\sigma_{0}\right)^{-1}} E_{0}^{r}$ $+2 M^{A+2}\left(\sigma_{2}-\sigma_{0}\right)\left(2\left(1+\left(\log \left(\frac{D}{\sigma_{1}-\sigma_{0}}\right)\right)^{*}\right)\right)^{\left(\sigma_{2}-\sigma_{1}\right)\left(\sigma_{2}-\sigma_{0}\right)^{-1}}\left(\frac{2 E_{0}}{D}\right)^{r}$ where

$$
\begin{gathered}
E_{0}=\exp \left(\frac{\left(\sigma_{2}-\sigma_{1}\right)\left(\sigma_{1}-\sigma_{0}\right)}{\sigma_{2}-\sigma_{0}}\right), \\
I_{0}=\int_{|v| \leq D}\left|f\left(\sigma_{0}+i t_{0}+i v\right) \frac{d v}{\sigma_{0}-\sigma_{1}+i v}\right|, \\
I_{2}=\int_{|v| \leq D}\left|f\left(\sigma_{2}+i t_{0}+i v\right) \frac{d v}{\sigma_{2}-\sigma_{1}+i v}\right|,
\end{gathered}
$$

and we have written $x^{*}=\max (0, x)$ for any real number $x$.

Remark. We have borrowed the result with $C=1$ from our paper [3].

In the rest of this section we state a conjecture which gives a lower bound for the mean-value of Titchmarsh series. We then collect some special results where the conjecture is proved. We borrow Sections 2 and 3 of [2]. We believe that the following conjecture is true (at least in a modified form). We stipulate that certain constants shall be integers only for a technical reason which is not serious.

Conjecture. Let $1=\mu_{1}<\mu_{2}<\ldots$ be any sequence of real numbers with $C^{-1} \leq \mu_{n+1}-\mu_{n} \leq C$, where $C(\geq 1)$ is an integer constant and $n=1,2,3, \ldots$ Let us form the sequence $1=\lambda_{1}<\lambda_{2}<\ldots$ of all possible distinct finite power products of $1=\mu_{1}<\mu_{2}<\ldots$ with non-negative integral exponents. Let $s=\sigma+i t, H(\geq 10)$ a real parameter and $\left\{a_{n}\right\}$ $(n=1,2,3, \ldots)$ with $a_{1}=1$ be any sequence of complex numbers (possibly depending on $H)$ such that $F(s)=\sum_{n=1}^{\infty} a_{n} \lambda_{n}^{-s}$ is absolutely convergent 
at $s=B$ where $B(\geq 3)$ is an integer constant. Suppose $F(s)$ can be continued analytically in $(\sigma \geq 0,0 \leq t \leq H)$ and that there exist $T_{1}, T_{2}$, with $0 \leq T_{1} \leq H^{3 / 4}, H-H^{3 / 4} \leq T_{2} \leq H$, such that for some $K(\geq 30)$

$$
\max _{\sigma \geq 0}\left(\left|F\left(\sigma+i T_{1}\right)\right|+\left|F\left(\sigma+i T_{2}\right)\right|\right) \leq K \text {. }
$$

Finally, let $\sum_{n=1}^{\infty}\left|a_{n}\right| \lambda_{n}^{-B} \leq H^{A}$ where $A(\geq 1)$ is an integer constant. Then there exists a $\delta_{1}(>0)$ (depending only on $\left.A, B, C\right)$ such that for all $H \geq H_{0}(A, B, C)$

$$
\frac{1}{H} \int_{0}^{H}|F(i t)|^{2} d t \geq \frac{1}{2} \sum_{\lambda_{n} \leq H^{\delta_{1}}}\left|a_{n}\right|^{2}
$$

provided $H^{-1} \log \log K$ does not exceed a small positive constant.

R e m ark 1 . We have used the symbol $\delta_{1}$ (in place of $\delta$ ) so that it should not clash with the $\delta$ already introduced. Also we conjecture that $1 / 2$ can be replaced by a quantity $\sim 1$ provided $H^{-1} \log \log K \rightarrow 0$ as $H \rightarrow \infty$. Whenever we have succeeded in proving this conjecture we have proved it in this stronger form. by

Remark 2. We need this conjecture only for $a_{n}$ defined (for large $\sigma$ )

$$
F(s)=(G(1 / 2+\delta+s))^{1 / q}=\sum_{n=1}^{\infty} a_{n} \lambda_{n}^{-s}
$$

and for any fixed $\delta>0$ and a suitable rectangle $(\sigma \geq 0, T \leq t \leq T+H)$ where $G(1 / 2+\delta+s)$ has no zeros. We choose $q$ to be any integer $(\geq 2)$.

We now quote the corollaries to the main theorem of [4].

Theorem $2^{*}$. Let $\mu_{n}=n$. Then the conjecture is true.

TheOREM $3^{*}$. Let $n_{0}(\geq 2)$ be any integer constant and

$$
\mu_{n}=\left(n_{0}+n-1\right) n_{0}^{-1} \text {. }
$$

Then the conjecture is true.

THEOREM $4^{*}$. Let $\beta(>0)$ be any algebraic constant and

$$
\mu_{n}=(n+\beta)(1+\beta)^{-1} \text {. }
$$

Then the conjecture is true (the conjecture is also true for the choice $\mu_{1}=1$ and $\mu_{n}=n+\beta-1$ for $\left.n>1\right)$.

Remark. It is possible to state a more general corollary than Theorem $4^{*}$. But we do not state it since our ambition is to prove a sufficiently general result.

We now record two important observations as Lemmas $1^{*}$ and $2^{*}$. 
LeMma $1^{*}$. Let $\mu_{n}=\left(n_{0}+n-1\right) n_{0}^{-1}$ where $n_{0}(\geq 1)$ is an integer constant and $G(s)=1-\sum_{n=2}^{\infty} b_{n} \mu_{n}^{-s}$ be absolutely convergent for some complex s. Then we have, for any integer $q(>0)$ and all $\sigma$ large enough, and any $\delta>0$,

$$
G(1 / 2+\delta+s)=\sum_{n=1}^{\infty} a_{n} \lambda_{n}^{-s}
$$

where the $\lambda$ 's are formed as in the conjecture, $a_{1}=1$ and further whenever $n_{0}+n-1$ is prime, $\left|a_{n}\right|=q^{-1}\left|b_{n}\right| \mu_{n}^{-1 / 2-\delta}$ and so

$$
\sum_{\lambda_{n} \leq H^{\delta_{1}}}\left|a_{n}\right|^{2} \geq q^{-2} \sum_{\mu_{n} \leq H^{\delta_{1}}}^{\prime}\left|b_{n}\right|^{2} \mu^{-1-2 \delta}
$$

where the accent denotes the restriction of the sum to those $n$ for which $n_{0}+n-1$ is prime.

Proof. See Lemma 1 of [2].

Lemma $2^{*}$. Let $G(s)=1-\sum_{n=2}^{\infty} b_{n} \mu_{n}^{-s}$ where the $b_{n}$ are non-negative and the sum involved converges for some complex $s$. Then for any integer $q$ $(>0)$ and for all large $\sigma$ and any $\delta>0$

$$
(G(1 / 2+\delta+s))^{1 / q}=\sum_{n=1}^{\infty} a_{n} \lambda_{n}^{-s}
$$

where the $\lambda$ 's are formed as in the conjecture, $a_{1}=1$ and further for $n \geq 2$, $a_{n} \leq 0$ and $-a_{n} \geq b_{n} \mu_{n}^{-1 / 2-\delta} q^{-1}$ whenever $\lambda_{n}=\mu_{n}$ and so

$$
\sum_{\lambda_{n} \leq H^{\delta_{1}}}\left|a_{n}\right|^{2} \geq q^{-2} \sum_{\mu_{n} \leq H^{\delta_{1}}} b_{n}^{2} \mu_{n}^{-1-2 \delta} .
$$

Pr o of. Trivial.

3. An outline of the method. Instead of giving a detailed proof of Theorems 1, 2 and 3 we give a rough sketch of the proof. We begin with

Lemma 1. Let $t_{0} \geq 100$ and let $D(s)$ be any function analytic in $(\sigma \geq$ $\left.1 / 2+\delta,\left|t-t_{0}\right| \leq C(\delta)\right)$ where $\delta$ is any positive constant and $C(\delta)$ is a large positive constant depending on $\delta$ and $D_{0}$ to follow. In this region let the maximum of $|D(s)|$ be $\leq M(\geq 30)$ and also $D(s) \neq 0$. Suppose further that for all $\sigma$ exceeding a constant $D_{0}$ we have $|\log D(s)| \leq 1 / 2$. Then $\log D(s)=O(\log M)$ in $\left(\sigma \geq 1 / 2+3 \delta / 2,\left|t-t_{0}\right| \leq C(\delta) / 2\right)$ and $\log D(s)=O\left((\log M)^{\theta}\right)$, with a $\theta(<1)$ not depending on $t_{0}$ in $(\sigma \geq 1 / 2+2 \delta$, $\left.\left|t-t_{0}\right| \leq C(\delta) / 3\right)$. Here the $O$-constants depend on $\delta$ and $D_{0}$.

R e m a r k. For the purposes of the present paper the conclusion $\log D(s)$ $=o(\log M)$ will do in place of $O\left((\log M)^{\theta}\right)$. But we have stated the lemma in this form since we will need it in a later paper [5]. 
Proof. The proof is essentially due to J. E. Littlewood. See pages 336337 of [8] for a proof which can be easily generalised to give this lemma.

We introduce small positive constants $\varepsilon_{1}, \varepsilon_{2}, \varepsilon_{3}, \varepsilon$ and $\delta$ (all $\left.<1 / 100\right)$ and these will be fixed in a suitable way. The only free parameters will be $T$ and $H$ ( $H$ will be fixed to be a large constant in the end).

LEMma 2. Let $A(s)=\sum_{n \leq T^{\nu}} b_{n} \mu_{n}^{-s}$ where $b_{1}=-1, \mu_{1}=1 \nu=\mu / 2$ and $b_{n}$ as before. Then, for $\sigma \geq 1 / 2+\delta$,

$$
\int_{T}^{T+T^{\mu}}|A(s)|^{2} d t \leq T^{\mu}\left(1+\varepsilon_{2}\right) \sum_{n=1}^{\infty}\left|b_{n}\right|^{2} \mu_{n}^{-2 \sigma},
$$

for arbitrary $\varepsilon_{2}>0$ and all large $T$.

P r o of. The lemma follows by standard arguments.

Divide the interval $\left[T, T+T^{\mu}\right]$ into $N$ abutting $t$-intervals $J$ (ignoring a bit at one end) each of length $H$ where $N \sim T^{\mu} H^{-1}$. With any interval $J$ associate the interval $J_{1}$ obtained by deleting intervals of length $H^{1 / 2}$ at both ends. If the maximum of $|G(s)|$ taken over the rectangle $(\sigma \geq 1 / 2+2 \delta$, $t \in J_{1}$ ) is $\geq T^{\varepsilon}$, then by Lemma 1 (applied to $G(s)$ and by taking $M$ to be a large positive constant power of $T)$ the rectangle $(\sigma \geq 1 / 2+\delta, t \in J)$ must contain a zero of $G(s)$. Otherwise we are easily led to the contradiction $T^{\varepsilon}=O\left(\exp \left((\log T)^{\theta}\right)\right)$. If there are $\geq \varepsilon_{1} N\left(\varepsilon_{1}>0\right.$ a small constant $)$ such rectangles $\left(\sigma \geq 1 / 2+2 \delta, t \in J_{1}\right)$ where $\max |G(s)| \geq T^{\varepsilon}$ then there is nothing to prove, since each rectangle $(\sigma \geq 1 / 2+\delta, t \in J)$ will contain a zero and the total number of zeros would be $\geq \varepsilon_{1} N \gg T^{\mu} H^{-1}$. Hence we assume that the number of rectangles $\left(\sigma \geq 1 / 2+2 \delta, t \in J_{1}\right)$ where $\max |G(s)| \geq T^{\varepsilon}$ is $<\varepsilon_{1} N$. Hence for $\geq\left(1-\varepsilon_{1}\right) N\left(\sim\left(1-\varepsilon_{1}\right) T^{\mu} H^{-1}\right)$ such rectangles $\left(\sigma \geq 1 / 2+2 \delta, t \in J_{1}\right) \max |G(s)|<T^{\varepsilon}$. Denote the set of these rectangles by $S$. We prove that a (positive) constant proportion of these rectangles must contain a zero of $G(s)$. Denote by $J_{2}$ the $t$-intervals $J_{1}$ of $S$ with intervals of length $H^{1 / 2}$ deleted from both ends. For $t_{0} \in J_{2}$ we now apply convexity Theorem $1^{*}$ taking $A=1$ and $M$ to be a large (positive) constant power of $T ; r=[\varepsilon \log T], H$ large enough, $a=1 / 2+2 \delta, b$ a large (positive) constant independent of $\varepsilon$ and $\delta, \sigma_{0}=a, \sigma_{2}=b, b / 2 \geq$ $\sigma_{1} \geq 1 / 2+3 \delta, D=\sqrt{H}$ and $f(s)=(G(s))^{2}-(A(s))^{2}$ where $A(s)$ is as in Lemma 2. It follows that

$$
\left|2 \pi f\left(s_{1}\right)\right| \leq 2\left\{I_{0}^{\sigma_{2}-\sigma_{1}}\left(I_{2}+T^{-3}\right)^{\sigma_{1}-\sigma_{0}}\right\}^{\left(\sigma_{2}-\sigma_{0}\right)^{-1}} T^{\varepsilon}+T^{-3},
$$

where

$$
I_{0}=\int_{|v| \leq \sqrt{H}}\left|f\left(\sigma_{0}+i t_{0}+i v\right) \frac{d v}{\sigma_{0}-\sigma_{1}+i v}\right|
$$


and

$$
I_{2}=\int_{|v| \leq \sqrt{H}}\left|f\left(\sigma_{2}+i t_{0}+i v\right) \frac{d v}{\sigma_{2}-\sigma_{1}+i v}\right| .
$$

Notice that for rectangles of $S$ we have $|G(s)|^{2}<T^{2 \varepsilon}$. We now integrate (1) with respect to $t_{0} \in J_{2}$. Now as $t_{0}$ varies over $J_{2}, t_{0}+v$ runs over at most $J_{1}$. Thus we get from (1)

$$
\begin{aligned}
\frac{2 \pi}{\left|J_{2}\right|} \int_{t_{0} \in J_{2}}\left|f\left(s_{1}\right)\right| d t_{0} \\
\leq 2\left\{\left(\frac{1}{\left|J_{2}\right|} \int_{t_{0} \in J_{2}} I_{0} d t_{0}\right)^{\sigma_{2}-\sigma_{1}}\left(\frac{1}{\left|J_{2}\right|} \int_{t_{0} \in J_{2}} I_{2} d t_{0}+T^{-3}\right)^{\sigma_{1}-\sigma_{0}}\right\}^{\left(\sigma_{2}-\sigma_{0}\right)^{-1}}+T^{-3} \\
=O\left(\left(\frac{1}{H} \int_{t_{0} \in J_{1}}\left|f\left(\sigma_{0}+i t_{0}\right)\right| d t_{0}\right)^{\sigma_{2}-\sigma_{1}}\right. \\
\left.\quad \times\left(\frac{1}{H} \int_{t_{0} \in J_{1}}\left|f\left(\sigma_{2}+i t_{0}\right)\right| d t_{0}+T^{-3}\right)^{\sigma_{1}-\sigma_{0}}\right)^{\left(\sigma_{2}-\sigma_{0}\right)^{-1}}+T^{-3} .
\end{aligned}
$$

Hence

(4)

$$
\begin{aligned}
& \frac{1}{H} \int_{t_{0} \in J_{2}}\left|f\left(s_{1}\right)\right| d t_{0} \\
&= O\left(\left\{\left(\frac{1}{H} \int_{t_{0} \in J_{1}}\left|A\left(\sigma_{0}+i t_{0}\right)\right|^{2} d t_{0}+T^{2 \varepsilon}\right)^{\sigma_{2}-\sigma_{1}}\right.\right. \\
&\left.\left.\times\left(T^{C_{0}-\mu \sigma_{2} / 2}+T^{-3}\right)^{\sigma_{1}-\sigma_{0}}\right\}^{\left(\sigma_{2}-\sigma_{0}\right)^{-1}}\right)+O\left(T^{-3}\right) .
\end{aligned}
$$

By Lemma 2 we have

$$
\sum_{J_{1}} \int_{t_{0} \in J_{1}}\left|A\left(\sigma_{0}+i t_{0}\right)\right|^{2} d t_{0}<T^{\mu}\left(1+\varepsilon_{2}\right) \sum_{n=1}^{\infty}\left|b_{n}\right|^{2} \mu_{n}^{-1-4 \delta},
$$

and so

$$
\int_{t_{0} \in J_{1}}\left|A\left(\sigma_{0}+i t_{0}\right)\right|^{2} d t_{0}>H\left(1+\varepsilon_{2}\right)\left(1+3 \varepsilon_{2}\right) \sum_{n=1}^{\infty}\left|b_{n}\right|^{2} \mu_{n}^{-1-4 \delta},
$$

for at most $\leq\left(1+3 \varepsilon_{2}\right)^{-1} T^{\mu} H^{-1}$ intervals $J_{1}$. Hence for $\geq\left(1-2 \varepsilon_{1}\right) T^{\mu} H^{-1}-$ $\left(1+3 \varepsilon_{2}\right)^{-1} T^{\mu} H^{-1} \gg T^{\mu} H^{-1}$ (provided $\varepsilon_{2}=10 \varepsilon_{1}$ ) rectangles of $S$ we have 
$|G(s)|^{2}<T^{2 \varepsilon}$ and also $\int_{t_{0} \in J_{1}}\left|A\left(\sigma_{0}+i t_{0}\right)\right|^{2} d t_{0}=O(H)$. Thus from (4) we obtain (for $\gg T^{\mu} H^{-1}$ out of these rectangles of $S$ )

$$
\frac{1}{H} \int_{t_{0} \in J_{2}}\left|f\left(s_{1}\right)\right| d t_{0}=O\left(\left(T^{2 \varepsilon\left(\sigma_{2}-\sigma_{1}\right)} T^{-3\left(\sigma_{1}-\sigma_{0}\right)}\right)^{\left(\sigma_{2}-\sigma_{0}\right)^{-1}}+T^{-3}\right)
$$

by choosing $\sigma_{2}$ such that $C_{0}-\mu \sigma_{2} / 2 \leq-3$. Now choose $\varepsilon$ such that

$$
2 \varepsilon\left(\sigma_{2}-\sigma_{1}\right) \leq 3\left(\sigma_{1}-\sigma_{0}\right)-\varepsilon .
$$

Then we have

$$
\frac{1}{H} \int_{t_{0} \in J_{2}}\left|f\left(s_{1}\right)\right| d t_{0}=O\left(T^{-\varepsilon\left(\sigma_{2}-\sigma_{0}\right)^{-1}}\right) .
$$

Again since $\left|G\left(s_{1}\right)\right|^{2} \leq\left|f\left(s_{1}\right)\right|+\left|A\left(s_{1}\right)\right|^{2}$ we have

$$
\frac{1}{H} \int_{t_{0} \in J_{2}}\left|G\left(s_{1}\right)\right|^{2} d t_{0} \leq\left(1+10 \varepsilon_{2}\right) \sum_{n=1}^{\infty}\left|b_{n}\right|^{2} \mu_{n}^{-2 \sigma_{1}},
$$

by Lemma 2 . Since the absolute value of an analytic function at a point is majorised by its mean-value taken over a disc of radius $\delta / 10$ about that point as centre, we see that the maximum of $|G(s)|$ taken over the rectangle $\left(\sigma \geq 1 / 2+3 \delta, t \in J_{3}\right)$ is $\leq H^{2}$ ( $J_{3}$ being $J_{2}$ with the intervals of length $H^{1 / 2}$ deleted at both ends). Hence by Theorems $2^{*}, 3^{*}$ and $4^{*}$ and Lemmas $1^{*}$ and $2^{*}$ we have

$$
\frac{1}{H} \int_{t_{0} \in J_{3}}\left|G\left(1 / 2+3 \delta+i t_{0}\right)\right|^{2 / q} d t_{0} \geq q^{-2}\left(1-\varepsilon_{3}\right) \sum_{\mu_{n} \leq H^{\delta_{1}}}^{\prime}\left|b_{n}\right|^{2} \mu_{n}^{-1-6 \delta}
$$

where the accent indicates the inclusion of only those $n$ for which $\left|a_{n}\right| \geq$ $q^{-1}\left|b_{n}\right| \mu_{n}^{-1 / 2-3 \delta}$. Also $\varepsilon_{3}$ is an arbitrarily small constant which is fixed. (We have applied the theorems and lemmas with $\delta$ replaced by $3 \delta$.) We compare this with the upper bound

$$
\left\{\left(1+10 \varepsilon_{2}\right)\left(\sum_{n=1}^{\infty}\left|b_{n}\right|^{2} \mu_{n}^{-1-6 \delta}\right)\right\}^{1 / q}
$$

obtained from (5) by Hölder's inequality. This leads to a contradiction (for $\gg T^{\mu} H^{-1}$ out of the rectangles of $S$ ) to our assumption of Theorem 2 (resp. Theorem 3). This completes the proof of Theorems 1, 2 and 3 stated in the introduction.

Acknowledgements. The authors are indebted to Professors M. Jutila, A. Ivić, D. R. Heath-Brown and B. C. Berndt for encouragement. 


\section{References}

[1] R. Balasubramanian and K. Ramachandra, On the zeros of a class of generalised Dirichlet series. III, J. Indian Math. Soc. 41 (1977), 301-315.

[2] - - - On the zeros of a class of generalised Dirichlet series. XI, to appear.

[3] - - - Some local convexity theorems for the zeta-function-like analytic functions, Hardy-Ramanujan J. 11 (1988), 1-12.

[4] - - - Proof of some conjectures on the mean-value of Titchmarsh series. III, Proc. Indian Acad. Sci. Math. Sci. 102 (1992), 83-91.

[5] -, - On the zeros of $\zeta(s)-a$, Acta Arith., to appear.

[6] B. C. Berndt, On the number of zeros of $\zeta^{(k)}(s)$, J. London Math. Soc. (2) 2(1970), $577-580$

[7] N. Levinson and H. L. Montgomery, Zeros of the derivative of the Riemann zeta-function, Acta Math. 133 (1974), 49-65.

[8] E. C. Titchmarsh, The Theory of the Riemann Zeta-function, 2nd ed. (revised and edited by D. R. Heath-Brown), Clarendon Press, Oxford 1986.

MATSCIENCE

THARAMANI P.O

MADRAS 600113

INDIA
SCHOOL OF MATHEMATICS

TATA INSTITUTE OF FUNDAMENTAL RESEARCH

HOMI BHABHA ROAD

COLABA

BOMBAY 400005

INDIA 